\title{
PARTICIPACIÓN DE LAS FAMILIAS INMIGRANTES Y EDUCACIÓN INTERCULTURAL EN LA ESCUELA
}

\author{
Juan José Leiva Olivencia \\ Universidad de Málaga
}

\begin{abstract}
RESUMEN: Este trabajo focaliza su mirada en la participación de las familias inmigrantes en el contexto escolar, analizando dicha participación como una iniciativa fundamental en la generación y desarrollo de una convivencia educativa intercultural en el marco de unas instituciones educativas que pretenden ser inclusivas. En este sentido, planteamos que la interculturalidad necesita de prácticas activas y democráticas como la participación comunitaria escolar en contextos educativos de diversidad cultural, ya que permite a los más jóvenes aprender modelos de relaciones y valores sociales positivos. En efecto, un reciente estudio de investigación realizado en centros educativos públicos de Primaria y Secundaria de la provincia de Málaga, confirma la tendencia creciente de considerar la importancia de promover la interculturalidad y la implicación de las familias inmigrantes para mejorar la construcción de una convivencia escolar intercultural e inclusiva.
\end{abstract}

ABSTRACT: This paper focuses its gaze on the participation of immigrant families in the school context, analyzing this participation as a key initiative in the generation and development of intercultural educational coexistence within the framework of educational institutions seeking to be inclusive. In this sense, we argue that multiculturalism requires active and democratic practices as the school community participation in educational settings of cultural diversity, and enabling more young people to learn models of relationships and positive social values. Indeed, a recent research study conducted in public schools Primary and Secondary Education in the province of Malaga, confirms the growing tendency to consider the importance of promoting intercultural and the involvement of immigrant families to improve the construction of a school life intercultural and inclusive.

PALABRAS CLAVE: Educación Intercultural, familias inmigrantes, diversidad cultural, convivencia, escuela inclusiva.

KEYWORDS: Intercultural Education, immigrant families, cultural diversity, coexistence, inclusive school. 


\section{INTRODUCCIÓN}

La escuela está afrontando el reto de la convivencia intercultural, y en nuestro país, las aulas han pasado en poco tiempo de ser espacios monoculturales a multiculturales (Esteve, 2004), y con la intención de aspirar a construir de manera cooperativa una educación intercultural que profundice en una escuela inclusiva. Aprovechar educativamente la diversidad cultural pasa por reconocer que lo común es la diversidad y que la diversidad debe ser, desde la perspectiva pedagógica, lo común en los diseños y propuestas de intervención educativa que se consideren, ya no sólo oportunos, sino imprescindibles en una sociedad democrática como la nuestra. La Educación Intercultural como propuesta pedagógica relevante en la educación actual tiene ante sí múltiples retos, uno de los cuales es consolidar su espacio conceptual propio, asumiendo la necesidad de fundamentar nuevas acciones pedagógicas como la promoción de la participación de las familias inmigrantes en el desarrollo de la convivencia educativa intercultural e inclusiva.

El presente trabajo pretende contribuir a indagar en la idea de la interculturalidad como principio clave de la participación escolar, atendiendo a la importancia creciente de la participación comunitaria como valor socioeducativo de primer orden en las instituciones educativas. En este punto, pretendemos exponer algunos resultados de un proyecto de investigación realizado en distintos centros educativos públicos de la provincia de Málaga ${ }^{1}$, planteando la perspectiva de los distintos agentes de la comunidad educativa (profesorado, familias, alumnado, educadores/as sociales, mediadores interculturales, etc.) en relación a la participación de las familias inmigrantes en las escuelas donde se están desarrollando proyectos educativos interculturales.

\section{UNA INVESTIGACIÓN EN EDUCACIÓN INTERCULTURAL}

En los últimos años han proliferado numerosos estudios de investigación sobre interculturalidad y organización escolar, si bien, como plantea García Castaño y Otros (2008) habría que señalar que los temas de convivencia escolar que se dan en los contextos educativos interculturales, tienen unas características cuyo análisis y estudio han estado generalmente determinados por la reflexión de los distintos modelos comunicativos, de disciplina y de clima de aula, siendo frecuentes los estudios centrados en las actitudes y actividades que se esperan del docente ante la educación intercultural, y los propios modelos formativos adecuados para la formación del profesorado en cuestiones relacionados con la interculturalidad y la diversidad del alumnado. Es decir, a pesar de ser una temática ampliamente tratada en diferentes estudios e investigaciones, desde nuestra perspectiva resulta interesante indagar y profundizar más en la perspectiva de los docentes ante la convivencia intercultural, pues es una realidad ineludible que el escenario pre-

1. Datos extraídos del trabajo de Leiva (2007), titulado "Educación y conflicto en escuelas interculturales", así como del Proyecto de Excelencia HUM1549, financiando por la Consejería de Innovación, Ciencia y Empresa de la Junta de Andalucía, realizado en el marco del Grupo HUM-169 de Teoría de la Educación y Educación Social de la Universidad de Málaga, dirigido por los profesores J. M. Esteve y J. Vera. 
sente y futuro de nuestras aulas y escuelas va a seguir siendo intercultural. Por otro lado, es necesario expresar que muchas de las investigaciones realizadas en estos últimos años, tanto en nuestro país como en la Unión Europea, han sido de corte cuantitativo y estadístico, las cuales se revelaban como insuficientes para una comprensión más profunda de la realidad educativa intercultural (Banks, 2008). Frente a ello, es necesario mencionar que la investigación cualitativa ha alcanzado en estos años el reconocimiento de configurarse como una vía rigurosa para acceder al conocimiento científico (Stake, 1998), y que en el campo de la educación intercultural es creciente su empleo como método y planteamiento de coherencia investigadora. Ejemplos de diseños investigadores de esta índole lo encontramos en diversos autores (Montón, 2004; Santos, 2008; Soriano, 2008) que además plantean la necesidad de conjugar diferentes enfoques metodológicos en el marco de un paradigma hermenéutico-interpretativo de investigación. En este sentido, a raíz de la importancia creciente de la relación entre los conceptos de convivencia, diversidad cultural, interculturalidad y participación escolar decidimos preguntar a profesores, alumnos y familias sobre su concepción pedagógica acerca de la participación de las familias inmigrantes como elemento de análisis clave en la generación de la convivencia intercultural en los centros escolares. Y, para intentar aportar elementos de análisis y reflexión sobre ello, expondremos algunos resultados de la de investigación cualitativa realizada al respecto en distintos centros educativos públicos de Málaga (Leiva, 2007), en la que analizamos la perspectiva de los distintos agentes de la comunidad educativa (profesorado, familias, alumnado, educadores/as sociales, mediadores interculturales, etc.) en relación a la participación de las familias inmigrantes en las escuelas donde se están desarrollando proyectos educativos interculturales, así como propuestas para mejorar la formación docente en participación comunitaria. Tenemos que subrayar que nos situamos en el paradigma interpretativo de investigación desde el punto de vista epistemológico, y utilizamos como instrumentos de recogida de datos cualitativos fundamentalmente las entrevistas en profundidad, observaciones, diarios investigadores y recopilación documental. Como procedimiento de análisis cualitativo usamos procesos de segmentación y categorización temática, con la ayuda del programa informático de análisis de datos textuales Nudist-Vivo, atendiendo a los criterios de solidez y coherencia interna de metodología cualitativa necesarios en este tipo de investigación (Sandín, 2003).

Por tanto, el objetivo fundamental de nuestra investigación era conocer y comprender las percepciones que tienen los distintos miembros de la comunidad educativa sobre la potencialidad de la participación familiar como instrumento pedagógico-intercultural. Como objetivos específicos planteábamos también conocer el grado de participación de las familias inmigrantes en la vida y convivencia educativa intercultural en los centros escolares; identificar las preocupaciones e inquietudes del profesorado en relación a la participación de las familias inmigrantes y su vinculación con la convivencia escolar entre alumnos de diferentes culturas en sus centros escolares; $y$, finalmente, comprender las concepciones e ideas educativas de los diferentes agentes educativos (profesorado, familias, alumnado) ante la participación de las familias autóctonas e inmigrantes. 


\section{PARTICIPACIÓN Y EDUCACIÓN INTERCULTURAL EN LA ESCUELA: ALGUNOS RESULTADOS DE INVESTIGACIÓN}

Tal y como ponen de manifiesto diferentes estudios realizados al respecto (Leiva 2005 y 2008; Santos, 2008; Santos, Lorenzo y Priegue, 2011), las familias inmigrantes tienen depositada una gran confianza en la escuela como vehículo de integración, tanto individual como familiar, en la sociedad de acogida. Esto lo hemos podido confirmar en un último estudio realizado² (Leiva, 2007): las familias inmigrantes tienen expectativas positivas de la educación que reciben sus hijos en la escuela pública. Además, participan cada vez más en la vida educativa de las escuelas a las que acuden sus hijos, a pesar de los condicionantes laborales y sociales que pueden determinar su participación en el contexto escolar.

"... (hay que) hacer e intentar acercar a estas madres al colegio, (...), que participen más en el colegio, que vean cuales son las actividades que sus hijos necesitan, cual es la necesidad de sus hijos dentro del colegio, (...), hay que aprovechar esa riqueza cultural de cada una de las nacionalidades..." (Fragmento de Entrevista a Mediadora Intercultural $)^{3}$.

En realidad, si partimos de la idea de que el ambiente familiar y escolar son los que más influyen en el desarrollo de la persona y su proceso educativo, no cabe duda de que entre escuela y familia debe existir una estrecha comunicación, a fin de lograr una visión globalizada y completa del alumno, reduciendo en la medida de lo posible, discrepancias en favor de la convergencia de criterios de actuación, apoyo mutuo y valores educativos.

En nuestra investigación, desarrollada en centros educativos públicos de educación primaria y secundaria de la provincia de Málaga, la participación de las familias inmigrantes es vista por los docentes como un instrumento fundamental para la construcción de la interculturalidad. Para promover una educación verdaderamente intercultural es necesario generar un nuevo espacio social de respeto a las diferencias humanas y sociales desde una perspectiva de igualdad y de inclusión para todas las personas y culturas (Bartolomé y Cabrera, 2003); y en verdad, esa construcción debe partir desde la participación de las familias en las escuelas, por lo cual, resulta decisivo facilitar y promover las condiciones óptimas para su participación efectiva. Ciertamente, podemos afirmar que las familias inmigrantes mues-

2. Tenemos que destacar el carácter eminentemente cualitativo del presente trabajo, que emplea entrevistas en profundidad, observaciones, diarios investigadores y recopilación documental como procedimientos de recogida de información a distintos miembros de la comunidad educativa (profesorado, madres y padres, alumnado, mediadores interculturales, educadores sociales y orientadores). Esta investigación se ha realizado en 8 centros educativos públicos de la provincia de Málaga (4 CEIPs y 4 IES), y se efectuaron 65 entrevistas. Por otra parte, decir que hemos empleado el análisis cualitativo de la información a través de procesos de categorización temática, con la ayuda del programa informático de análisis de datos textuales Nudist-Vivo 2.0.

3. Todos los nombres empleados en este trabajo son pseudónimos a fin de garantizar el anonimato de las personas que participaron en el mismo. 
tran un interés y preocupación relevantes por la educación que reciben sus hijos, por lo que su relación con el profesorado es cada vez más estrecha.

"...sabes que la familia inmigrante lo que quieren es mejorar..., ellos vienen huyendo de..., o buscando una mejor vida.., entonces cuando es una familia, cuando tienen hijos, pues ellos quieren unas expectativas, y unas aspiraciones de sus hijo de que estudien, de que estudien lo máximo... y es verdad que las familias inmigrantes se preocupan mucho de que estudien, están muy pendientes, y van a hablar mucho con los profesores..., porque claro, ya que están en España, porque saben que es un país es avanzado donde la educación es importante, y les interesa..." (Entrevista a Profesora de Primaria).

Compleja es siempre la tarea de llevar a la práctica estas ideas de mejora de la participación activa de las familias inmigrantes en la escuela, pues no en vano, una de las dificultades que manifiestan los profesores de los centros educativos que escolarizan a alumnos hijos de familias inmigrantes es encontrar los instrumentos de comunicación adecuados para estas familias, atendiendo a diferentes variables como incompatibilidad de horarios y de índole labora, incomunicación por desconocimiento del idioma, incertidumbre social en el entorno familiar, problemas económicos, falta de información u otros aspectos. Sin embargo, hemos descubierto la importancia de la empatía y la confianza mutua como herramientas claves en la relación que se puede establecer entre el profesorado y las familias inmigrantes. Escuchar al otro, comprenderlo y sentir que existe en la escuela un apoyo emocional además del educativo, resulta de gran trascendencia para el fomento de una actitud positiva en las familias respecto a la escuela. A fin de superar obstáculos y dificultades como los que acabamos de exponer, podemos decir que la planificación y desarrollo de un plan de acogida -o proyecto educativo intercultural- facilitaría la creación de espacios formativos alternativos y flexibles. En este punto, debemos afirmar que ya hoy tenemos claros ejemplos de escuelas inclusivas en las que se están llevando a cabo acciones educativas interculturales tendentes a promover la participación activa de las familias inmigrantes como un eje fundamental en la mejora de la convivencia en estos centros escolares (Casanova y Rodríguez, 2009).

Cuando hablamos de espacios formativos flexibles, nos estamos refiriendo a la cada vez más necesaria implantación de acciones educativas creativas donde la interculturalidad sea vivida y construida por todos los agentes de la comunidad educativa. Propuestas de este tipo son visibles en algunos centros que hemos estudiado. ¿Cuáles son estas propuestas? Los grupos de madres, las charlas con café, los encuentros educativos interculturales, los talleres extraescolares para alumnos y familias. En definitiva, nos estamos refiriendo a todas aquellas propuestas que dan cobertura a una estrategia consciente de reconocer la importancia de la participación de todas las familias (inmigrantes y autóctonas) para ir construyendo una escuela viva y optimista: la escuela de la diversidad.

Además, tenemos que señalar que las AMPAs tienen que jugar un papel clave en la gestión educativa de la diversidad cultural, y ahí es necesario que los equipos directivos apuesten decididamente por la dinamización de estos organismos como canalizadores de actividades educativas interculturales. Las escuelas de padres y madres, los encuentros informales, los espacios formativos de apoyo sobre el apren- 
dizaje de la lengua materna, los talleres (de temáticas interesantes y atractivas para las familias) o jornadas culturales diversas, pueden constituirse en nuevas herramientas educativas interculturales si el objetivo es lograr la inclusión educativa de todos sin excepciones y la mejora de la convivencia en las escuelas interculturales.

De nuestro estudio podemos extraer la idea de que las familias necesitan crear nuevos espacios de relación y un clima de diálogo activo con el apoyo de los docentes. No se trata sólo de poner a disposición los espacios y los tiempos -generalmente extraescolares-, sino, sobre todo, de promover una participación activa que movilice todos los recursos educativos disponibles y enfoque globalmente a la escuela como un escenario formativo comunitario, de servicio e interés público para toda la comunidad. Por esta razón, el profesorado de estas escuelas debe estar formado y sensibilizado en una educación intercultural democrática y participativa, y es que la escuela "es un lugar donde padres y profesores tienen que trabajar educación en valores, porque se pretende formar a niños con valores, no solamente a leer y a escribir" (Fragmento de Entrevista a Directora de CEIP).

Es necesaria la participación docente en los grupos de madres de origen inmigrante y en las actividades dirigidas a las familias que existen en los centros educativos. De hecho, hemos observado que estas plataformas educativas son muy importantes para avanzar en un diálogo enriquecedor e intercultural entre las propias familias inmigrantes y las autóctonas, ya que "crean redes de apoyo por ese sentimiento de desarraigo que algunas madres traen, y como ven a otras en características similares, pues se relacionan". En este sentido, consideramos que estas actividades dirigidas a madres inmigrantes son fundamentales para "fomentar los lazos sociales e incluso para que hagan amistades entre ellas mismas, ya que muchas madres necesitan mucho afecto y apoyo" (Fragmento de Entrevista a Profesora de ATAL). Desde nuestro punto de vista, la participación de las familias tiene un doble valor. No sólo es una acción educativa que promueve la mejora de las relaciones entre los alumnos de las diferentes culturas y, por tanto, la mejora de la convivencia en la escuela; también sirve como elemento de apoyo emocional para muchas familias que viven momentos vitales complejos y difíciles.

Que las familias sean agentes educativos activos y comprometidos con los principios pedagógicos de los centros escolares donde acuden sus hijos es una clave de enorme potencialidad para la gestión positiva de todos aquellos conflictos o situaciones de dificultad que puedan darse en el ámbito educativo. No obstante, la cuestión se plantea en si es o no efectiva, reflexiva y crítica la participación de las familias inmigrantes en el escenario escolar, y cuáles son los mecanismos que incentivan y dinamizan esa participación familiar. En nuestro estudio encontramos respuestas diversas a este aspecto de gran interés para la convivencia escolar. Por un lado, hay quienes afirman que la participación familiar en la escuela es cada vez mayor y tiene una repercusión significativa, mientras que otros consideran que es escasa y no tiene relevancia para la convivencia escolar. En todo caso, esas diferencias también se sitúan cuando la cuestión radica en la participación de las familias inmigrantes y autóctonas, es decir, hay centros donde las familias más activas son las inmigrantes mientras que en otros contextos socioeducativos, son las familias autóctonas las más participativas en el escenario escolar. 
Lo que sí podemos confirmar es que la valoración que hace el profesorado de estos centros hacia las familias inmigrantes es muy positiva, ya que se observa una actitud positiva de estas familias hacia la escuela y el propio sistema educativo de nuestro país. Esto, a juicio de algunos docentes, tiene algunas excepciones, y es que no se daría de manera generalizada en todos los grupos culturales, sino especialmente en algunas comunidades de familias inmigrantes como las de origen chino o de los países del este europeo, frente a las familias de origen latinoamericano o magrebí. Así se expresa un profesor de educación secundaria al respecto:

"Entrevistadora: Seguimos un poco con la relación entre las familias y la escuela. De cara a la participación real en las reuniones en las que habéis convocado a los padres, ipiensas que participan en la vida escolar los padres de un alumno inmigrante en la misma medida que los padres de un alumno autóctono?

Profesor: Yo creo que los padres de los alumnos inmigrantes tienen una actitud de valoración especial. Especial porque tienen una actitud de valorar el país que a lo mejor nosotros no tenemos. Es decir, ellos valoran mucho la oportunidad que les brinda a ellos y a su familia este país. Eso se manifiesta en una actitud, que uno observa, que mantiene el alumno por el hecho de estar escolarizado. Pero, jatención! Hay diferencia entre dos tipos de comunidades: no es lo mismo un chino que un alumno ucraniano, que un alumno marroquí, que un alumno de origen hispano americano, o de origen centroeuropeo." (Fragmento de Entrevista a Profesor de Primaria).

En cualquier caso, la participación de las familias inmigrantes en la escuela tiene cada día un mayor peso e influencia en el contexto escolar, sobre todo cuando son invitadas a algunas actividades donde se valora la diversidad cultural como un aspecto positivo y enriquecedor para la convivencia escolar. Por ejemplo, en las semanas o jornadas interculturales, el profesorado y la comunidad educativa en su conjunto invita a las familias inmigrantes para que participen en estas actuaciones que están contempladas en los proyectos educativos de los centros que acogen a un número significativo de alumnado de origen inmigrante. Además, la participación de estas familias es un aspecto muy valorado por el profesorado de estos centros escolares:

"Entrevistadora: Los padres de los niños inmigrantes ¿creen que participan en la vida del colegio más o menos igual que los padres de los niños españoles?

Profesor: Sí, de hecho el año pasado nos llevamos una sorpresa con la jornada intercultural porque teníamos dudas de que respondieran tan bien y salió una cosa muy especial." (Fragmento de Entrevista a Profesor de Secundaria).

Pero la participación de las familias inmigrantes no sólo se limita a determinados días o semanas del curso escolar, en algunas actuaciones denominadas interculturales, sino que tienen ya una mayor relevancia en el contexto educativo. En efecto, las madres y padres inmigrantes comienzan a participar en los órganos de representación y gestión de la vida escolar, instancias relevantes para la gestión positiva de la convivencia escolar, como el Consejo Escolar, donde las familias inmigrantes tienen ya un peso significativo. Así lo plantea una profesora:

"Entrevistador: ¿Los padres de los alumnos inmigrantes se implican en el centro en la misma medida que los padres de los alumnos autóctonos? 
Profesora: Sí, en este centro sí... de hecho en el Consejo Escolar tenemos representación de padres de niños inmigrantes, y en el Instituto no sé". (Fragmento de Entrevista a Profesora de Secundaria).

También hemos podido constatar en la investigación que, en general, las familias juegan un papel importante en el establecimiento del clima de convivencia escolar. Y algunas veces el profesorado percibe que las situaciones de conflicto escolar que plantean algunos alumnos en términos de conductas disruptivas tienen que ver con la situación familiar del alumno:

"Entrevistadora: Me refiero más bien a los padres de los niños que han tenido conflicto. Los padres de los niños que han tenido conflicto ijuegan algún papel en la solución de los conflictos?

Profesora: Hombre, si se ve que ya hay algún conflicto grande pues entonces el director o la orientadora ya tienen que hablar con los padres. Hablan con ellos. Hablan con la madre. A lo mejor son conflictos con ellos, con los padres. Muchas veces los conflictos son con los padres, claro. A lo mejor los niños con sus padres no se llevan bien. $O$ a lo mejor no hay buen clima en su casa. $Y$ si hay peleas en su casa en el Instituto no se puede pretender que sean buenos". (Fragmento de Entrevista a Profesora de Secundaria).

Los alumnos son también conscientes de las dificultades que implica la participación familiar en el contexto escolar, no tanto por las limitaciones que impone dicho contexto, sino por las diferentes situaciones de dificultad que pueden tener las familias a la hora de participar activamente en el escenario escolar. Estamos haciendo referencia a limitaciones horarias, sobre todo por cuestiones laborales, que muchas veces hacen incompatible la participación de las familias en los centros educativos. En el caso concreto de las familias inmigrantes, el tema laboral es una situación, si cabe más importante, ya que vienen a trabajar y ocupan su jornada diaria en su contexto laboral, lo que impide que muchas familias no puedan participar de manera efectiva en la escuela por incompatibilidad de horario o por el propio cansancio que implica una larga jornada laboral. Esta es la percepción que nos ofrece una alumna inmigrante:

"Entrevistadora: Entonces, si no vienen, claro, tampoco sabes si vienen los padres inmigrantes más que los de aquí...

Alumna Inmigrante: Los padres inmigrantes se preocupan... por ejemplo, en mi edificio son todos paraguayos y colombianos... y los padres se preocupan de hacer plata, se preocupan de eso, no se ponen a preocuparse por los estudios de sus hijos. Se preocupan o más por la economía familiar que por la educación de sus hijos. Si pueden hacer que trabaje más dinero entra en la casa." (Fragmento de Entrevista a Alumna Inmigrante de Secundaria).

Esta percepción es compartida por algunas madres de origen inmigrante, y es que no solamente existen dificultades de acceso a la participación familiar en la escuela por tema laboral, sino que también existe entre la población inmigrante una mentalidad poco propicia o abierta para la participación. Es decir, que por miedo o desconocimiento muchas familias temen participar de manera efectiva en la comunidad educativa, por lo que solamente se acercan a la escuela a preguntar por el ren- 
dimiento académico de su hijo y poco más. En efecto, podríamos hablar de unas barreras de mentalidad que hacen referencia a una dificultad comunicativa para la óptima participación de las familias inmigrantes en el contexto educativo. Y en el caso concreto de la relación educativa entre familias autóctonas e inmigrantes, una madre inmigrante nos aporta una interesante reflexión. Nos plantea que las barreras de comunicación entre ambas partes existen y que son más las barreras que ponen los inmigrantes que los autóctonos, lo cual es un elemento negativo si lo que se pretende es ofrecer a los alumnos un modelo positivo de relaciones interculturales, clave fundamental para el desarrollo práctico de la educación intercultural:

"Entrevistadora: Sí es verdad que he visto a alguna madre. Sí que tú dices que nos tendríamos que hacer una piña por estar aquí todos de fuera.

Madre Inmigrante: Exacto. Aquí la gente es muy abierta.

Entrevistadora: Pues yo me alegro de que estés contenta.

Madre Inmigrante: Sí, absolutamente.

Entrevistadora: Se te nota. ¿Y la relación que tenéis con la escuela? Es decir, los padres de alumnos inmigrantes tú ves que participan tanto como los de padres españoles, más, menos. ¿Cómo ves tú esa relación?

Madre Inmigrante: Los que más participan vuelvo a decirte son los españoles, los padres españoles, incluso organizan cosas para nosotros. Nosotros estamos todavía un poquito cerrados. Yo creo que hay más barreras puestas por parte de los que venimos que por parte de los que nos reciben en el caso especial de España. He emigrado a otros países también, estuve en Italia y era absolutamente diferente.

Entrevistadora: Diferente.

Madre Inmigrante: Estuve seis años en Italia y es totalmente diferente.

Entrevistadora: Te llama la atención el esfuerzo que se hace desde aquí para que vengáis.

Madre Inmigrante: $Y$ este centro, no conozco otro porque tengo un niño de cinco años. Que todavía no estoy muy en el tema escolar, que recién empieza". (Fragmento de Entrevista a Madre Inmigrante de CEIP).

Es posible que, al margen de las dificultades comunicativas entre familias, en el caso de las familias inmigrantes podamos observar una concepción más reduccionista de la participación de las familias en el escenario escolar. Y esto puede deberse a que muchas familias inmigrantes proceden de países donde existe una tradición escolar muy cerrada a la promoción de la participación democrática y activa de las familias en la escuela. Es decir, es posible que muchas familias inmigrantes tengan una concepción tradicional de la escuela, donde el profesorado es el protagonista absoluto de todas aquellas actuaciones y hechos que acontecen en la realidad escolar. Así lo considera una madre inmigrante al ser preguntada por los condicionantes de la participación real y efectiva de las familias inmigrantes en la escuela:

"Es que tenemos una tradición escolar muy cerrada con respecto a la familia. Entonces generalmente los padres no conocemos como es la vida en la escuela, como es el trabajo del docente. Y creo que todo esto ayuda a que todo esto sea más abierto, a que nos podamos integrar y colaborar, a que tengamos más relación con el traba- 
jo del docente y a lo mejor de esta manera también podamos llegar a valorarlo un poco más". (Fragmento de Entrevista a Madre Inmigrante de CEIP).

La cuestión de la participación de las familias inmigrantes en la escuela no es un tema menor en la convivencia escolar y en el desarrollo de la interculturalidad, más bien al contrario, es un tema central entre los principios pedagógicos de la educación intercultural y de la cultura de la diversidad en la escuela inclusiva. En este sentido, buena parte del análisis de la convivencia intercultural que hemos realizado en el estudio cualitativo que presentamos tiene su razón más intensa y profunda en la relación e interacción intercultural entre familias inmigrantes y autóctonas, y su participación en la escuela. En efecto, esto tiene que ver con diferentes modelos de integración cultural, es decir, con un modelo asimilacionista donde las familias inmigrantes se integran en el ámbito escolar excluyendo su identidad cultural en las acciones educativas, o por el contrario, un modelo de inclusión de la diversidad cultural donde todas las familias tienen una voz relevante para aportar soluciones y experiencias para avanzar hacia una convivencia intercultural.

También, tal y como plantea una madre autóctona, es posible la existencia de un modelo de autoexclusión cultural (segregacionista), donde las familias inmigrantes no tienen el menor interés en integrarse de manera efectiva en el contexto socioeducativo. No obstante, esto es un aspecto claramente discutible, ya que es posible que el problema parta de la sociedad de acogida, y por tanto de la comunidad educativa mayoritaria (autóctona), al plantear un modelo de relaciones interculturales supeditado a la asimilación cultural por parte de los inmigrantes, o en su defecto, un planteamiento fundamentalmente folclórico de las relaciones culturales con las negativas consecuencias que puede tener esto para la convivencia escolar:

"Madre Autóctona: No. jFinlandeses! Hay una colonia de finlandeses grandísima aquí, pero los ves que se van metiendo en las fiestas populares, se van metiendo, en fin.

Entrevistador: Sí.

Madre Autóctona: En fin, pero participan más. Principalmente intentan aprender el idioma, que eso es muy importante porque la verdad, si te cierras... Claro que en estos sitios como Fuengirola que es un sitio costero hay tiendas inglesas, comercios ingleses, bares ingleses. Entonces claro, no se calientan la cabeza. Y dirán: "pues yo para que me voy a calentar la cabeza." (Fragmento de Entrevista a Madre Autóctona de IES).

Y, por supuesto, la cuestión lingüística es un elemento clave cuando lo que estamos analizando es la participación de las familias inmigrantes en la comunidad educativa. En el caso de los inmigrantes no hispanoparlantes, ocurre al igual que sus hijos, esto es, iniciales dificultades de comunicación que pueden entorpecer la plena inclusión escolar de estas familias. En este punto, es digno de mencionar las dificultades que encuentran muchas madres inmigrantes para aprender el idioma, lo que implica una interesante reflexión de la profesora de un centro escolar. Esta docente nos plantea las dificultades de participación derivadas de la problemática laboral, unida a las dificultades de índole lingüística, lo que a su juicio requiere un esfuerzo extra por parte del profesorado para fomentar aún más si cabe un talante 
de apertura y de cercanía hacia estas familias, lo cual es un factor positivo para la convivencia escolar:

"O sea las madres marroquíes que acaban de salir de la reunión, son madres que ya hablan español como tú o como yo y sus hijos son muy españoles, o sea, ya están muy integradas. Entonces ellas también están más abiertas. Hay otros que por su situación, que la mayoría, sinceramente, la mayoría, porque aquí hay un tanto por ciento de gente que vive aquí por el sol y otros que vienen, la mayoría, pues a trabajar y la verdad es que no lo pasan muy bien al principio porque no pueden... Que pasa que muchos no pueden dedicar el tiempo a lo mejor a relacionarse tanto en el cole porque tienen que trabajar muchísimas horas. Eso es lo que a veces me dicen a mí ellos mismos cuando he tenido contacto con alguno. Que les gustaría a lo mejor tener más tiempo y el tiempo... Pero en general, la verdad es que este colegio, por lo que yo veo... Eso también el ambiente lo crean los profesores, ¿eh?, algunas veces". (Fragmento de Entrevista a Profesora de Primaria).

Esta idea es repetida por muchos docentes, es decir, la imposibilidad que tienen en muchas ocasiones de interactuar con las familias inmigrantes, incluso para tratar la evolución y el rendimiento académico de sus hijos. En este sentido, el profesorado es consciente de que los padres de los alumnos inmigrantes trabajan y tienen muchas dificultades para venir al centro educativo:

"Los padres de los alumnos inmigrantes que yo tengo ni siquiera los conozco. Están todo el día trabajando, tanto si hay padre como si no hay padre, porque algunos solamente vienen con la madre. Están siempre trabajando no tienen tiempo para venir." (Fragmento de Entrevista a Profesor de Secundaria).

Otro aspecto importante de análisis en la participación de las familias inmigrantes tiene que ver con el desarrollo de su identidad cultural en la sociedad de acogida. $Y$ es que, en muchas ocasiones, el profesorado viene observando como los inmigrantes de segunda generación tienen una actitud más positiva respecto a su propio papel como familias implicadas en una comunidad educativa, y por tanto, interesadas en participar activamente en la realidad escolar de los centros donde estudian sus hijos. Esto hace que, en algunos centros, la participación de familias inmigrantes sea superior que las autóctonas, lo que indica que este factor de índole sociocultural tiene una repercusión relevante para la convivencia escolar, y por supuesto, en las medidas para el desarrollo práctico de la educación intercultural en colegios e institutos:

Entrevistadora: Eso lo dicen mucho, que son lo que tú decías, son los reyes de la casa. ¿Participan igual los padres de niños inmigrantes en la vida escolar que los padres...?

Profesor: Sí, sí, sí. Y además hay muchos interesados en eso. Indudablemente los primeros años, tú sabes que la inmigración, la primera generación es de integración. Cuando realmente ya se encuentran totalmente integrados en la segunda generación es cuando ya se sienten totalmente del país. Vienen a las reuniones, vienen y no hay ninguna... Incluso en un porcentaje, según el número de alumnos, mayor que el de españoles, ¿eh? Porque a las reuniones vienen madres y muy pocos padres. (Fragmento de Entrevista a Profesor de Secundaria). 
De todas maneras, como hemos mencionado con anterioridad, la participación de las familias es un tema que modula entre diferentes consideraciones, tanto en el grado como en el protagonismo de quienes participan en el contexto escolar (inmigrantes y autóctonos). En este punto, tenemos que subrayar que nuestro equipo de investigación ha constatado la percepción generalizada de la existencia de una escasa participación por parte de las familias autóctonas, tanto desde la perspectiva de las familias inmigrantes como por parte de las mismas familias autóctonas. Es decir, que en centros educativos donde la diversidad cultural es una clave significativa en su realidad cotidiana, parece existir la idea de que las familias autóctonas participan menos que las inmigrantes, aunque en ocasiones se denunciaba la cierta inutilidad de la participación de las familias pues la autonomía de los centros se podía ver limitada por una normativa excesivamente reglada y burocratizada, lo que ofrece escasos incentivos a la efectiva y democrática participación de familias, tanto inmigrantes como autóctonas en el ámbito escolar:

"Además, es importante señalar la poca participación de las familias autóctonas tanto desde la perspectiva de los padres inmigrantes como desde la perspectiva de los padres autóctonos. Así, los padres autóctonos e inmigrantes que pudimos entrevistas eran los que participaban en el Consejo Escolar y ambos coincidían en señalar en que la participación en general de los padres en ese centro era baja. Los padres lamentaban esta poca participación paterna y denunciaban que muchos padres no participaban porque no podían (la educadora social del centro resaltaba cómo la situación de deprivación económica les dificultaba participar), pero que otros pasaban. Así mismo, los padres también denunciaban que la participación de los padres en los órganos de gestión del centro (claustro o comisión de convivencia) también se había visto limitada últimamente por las recientes normativas de la Junta de Andalucía." (Fragmento de Entrevista a Director de IES).

Los profesores lamentan la actitud pasiva de muchas familias en relación a su potencial papel protagonista en la realidad escolar. Esto se observa con preocupación en determinados grupos étnicos (gitanos, por ejemplo), y sobre todo en los contextos educativos en riesgo de exclusión social. En este punto, parece que las familias inmigrantes son más conscientes de la importancia de la educación de sus hijos, y de la necesidad de su participación real en los centros educativos.

Desde luego, el profesorado tiene una actitud positiva respecto a la importancia de contar con la colaboración y el apoyo de las familias en su tarea educadora. Es decir, los docentes valoran muy positivamente sentirse apoyados y respaldados en sus acciones docentes por las familias, lo que mejora la convivencia escolar y facilita un mejor rendimiento escolar del alumnado. Estas son las palabras del orientador de secundaria al ser preguntado por la importancia de la participación familiar en su instituto:

"...contar con el apoyo de la familia. Es decir, la participación y el apoyo de las familias es fundamental. Ante cualquier situación, ya sea positiva o negativa, tenemos que contar con ese apoyo familiar, que seguramente nos cuesta, pero que va a permitir conseguir un mayor resultado con el alumnado." (Fragmento de Entrevista a Orientador de Secundaria). 


\section{CONCLUSIONES FinAles}

De todo lo expuesto se desprende la idea de que las relaciones entre familias inmigrantes y autóctonas son todavía escasas, y solamente aquellas familias que tienen algún grado de amistad se relacionan de alguna manera. Las relaciones positivas entre familias de diferentes culturas deben ser un instrumento fundamental de interacción cultural de gran valor para los alumnos, pues supone un modelo de relaciones que favorece comportamientos prosociales y actitudes de apertura a la diversidad cultural. El problema es que todavía persiste cierto grado de rechazo de las familias autóctonas hacia la población escolar inmigrante, lo que tiene una implicación -emocional y social- directa en el sentido de excluir a estos alumnos y a sus familias, y por tanto, no favorecer las potencialidades de la diversidad cultural como factor de promoción de una convivencia educativa solidaria y tolerante.

Una lectura crítica de los datos obtenidos en nuestro estudio nos lleva a considerar que la participación de las familias, tanto inmigrantes como autóctonas, como de las organizaciones y entidades sociales, va a tener un papel cada día más relevante en el clima de convivencia escolar, sobre todo en los contextos educativos de diversidad cultural, que en términos generales, son más susceptibles de recibir apoyos, recursos y ayudas de diversa índole por parte de estas instituciones (ONGs, AA.VV.s, Fundaciones...). La cuestión clave es que el profesorado se mueve, a menudo, en un sistema de comunicación cerrado con el entorno social del centro educativo debido, en ocasiones, a la inseguridad que le suscita la falta de dominio de algunos temas de sensibilización y formación intercultural. Así mismo, es cierto que hemos constatado como hay algunos profesores que no aprovechan el potencial didáctico que le ofrecen muchas ONGs y grupos sociales que podrían colaborar en la transformación intercultural del curriculum escolar. En efecto, a pesar de que es cierto que cada día acuden con más frecuencia estas entidades a trabajar a la escuela, todavía persiste la idea de que la institución escolar no es el espacio natural para estas entidades sociales, las cuales acostumbran a basar su estrategia de acceso al centro escolar mediante la elaboración de materiales didácticos externos o bien la realización de algún tipo de actividad puntual -generalmente lúdica- en el contexto educativo formal. En todo caso, sí tenemos que confirmar que estas entidades no sólo trabajan en horario extraescolar, sino que ya actúan en el horario escolar, dotando de una gran vitalidad y participación democrática a la institución educativa, es decir, a unas escuelas que camina ya hacia la interculturalidad y que se constituyes en escuelas inclusivas (López Melero, 2006).

Nuestra propuesta va en la línea de construir una escuela intercultural entendida en términos de comunidad de aprendizaje, es decir, que el proyecto curricular de centro se haga no desde una perspectiva técnica o administrativa, sino fundamentalmente crítica y transformadora, con la participación activa e implicación crítica de alumnos, familias y entidades sociales en el marco de una comunidad intercultural de aprendizaje (Flecha, 2010; Valls y Otros, 2002). Se trataría de naturalizar la presencia y la relación educativa del profesorado con el resto de agentes de la comunidad educativa desde un enfoque de simetría participativa, es decir, que estas entidades y organizaciones sociales del barrio puedan concretar su representación y acción en la escuela a través de una comisión o un consejo que permita la actuali- 
zación curricular permanente. En definitiva, estamos planteando que es posible, y sobre todo necesaria, la transformación del curriculum escolar introduciendo nuevas opciones de participación comunitaria en la escuela. En este sentido, el empleo de las nuevas tecnologías y las comunidades virtuales son también dos caminos novedosos para iniciar y consolidar este tipo de participación escolar.

\section{REFERENCIAS BIBLIOGRÁFICAS}

BANKS, J. A. (2008). Diversity, Group Identity and Citizenship Education in a Global Age. Educational Researcher, 37 (3), pp. 129-139.

BARTOLOMÉ, M. (2002). Identidad y ciudadanía: un reto a la educación intercultural. Madrid: Narcea.

BARTOLOMÉ, M. y CABRERA, F. (2003). "Sociedad Multicultural y Ciudadanía: hacia una sociedad y ciudadanía interculturales". Revista de Educación, núm. extraordinario, pp. 33-56.

CASANOVA, M. A. y RODRÍGUEZ, H. J. (2009). La inclusión educativa, un horizonte de posibilidades. Madrid: La Muralla.

ESSOMBA, M. A. (2003). Educación e inclusión social de inmigrados y minorías. Tejer redes de sentido compartido. Barcelona: Cisspraxis.

ESSOMBA, M. A. (2007). "Estrategias de innovación para construir la escuela intercultural", en ÁLVAREZ, J. L. y BATANAZ, L. Educación intercultural e inmigración. De la teoría a la práctica. Madrid: Biblioteca Nueva, pp. 177-212.

ESTEVE, J. M. (2004). La formación del profesorado para una educación intercultural. Bordón, 56 (1), 95-115.

FLECHA, R. (2010). Prácticas educativas que fomentan la inclusión. Conclusiones del Proyecto Includ-ed. Actas del Simposio Diversidad Cultural y Escuela: El desarroIlo de la competencia intercultural. Badajoz: Universidad de Extremadura.

GARCÍA CASTAÑO, F. y otros (2008). Población inmigrante y escuela en España: un balance de investigación, Revista de Educación, 345, pp. 23-60.

LEIVA, J. (2005). "La participación comunitaria en la escuela como fundamento en la construcción de una sociedad intercultural", en PEIRÓ, S. Nuevos desafíos de la educación. Alicante. ECU (Vol. II), pp. 211-219.

LEIVA, J. (2007). Educación y conflicto en escuelas interculturales. Málaga: Spicum.

LEIVA, J. (2008). "La interculturalidad como principio clave de la educación para la ciudadanía: la participación de las familias inmigrantes en las escuelas interculturales", en SORIANO, E., y JIMÉNEZ, A. J. Ciudadanía, democracia y participación. Almería: Editorial Universidad de Almería, pp. 457-463.

LÓPEZ MELERO, M. (2006). Cultura de la diversidad, cultura de la inclusión: educar para construir una escuela sin exclusiones. En AA. VV., L'Ecola que inclou (pp.11-52), (Col-lecció Hort de Trenor 18). Torrent: Ajuntament de Torrent.

MONTÓN, M. J. (2004). La integración del alumnado inmigrante en el centro escolar. Orientaciones, propuestas y experiencias. Barcelona: Graó.

SANDíN, Ma . P. (2003). Investigación cualitativa en educación. Fundamentos y tradiciones. Madrid: McGraw-Hill Interamericana de España. 
SANTOS, M. A. (2008). "Las familias inmigrantes en la escuela. ¿Cómo avanzar en su integración desde una perspectiva socioeducativa?", en GARCÍA, J. y LACOMBA (Eds.). La inmigración en la sociedad española. Barcelona: Edicións Bellaterra, pp. 483-505.

SANTOS, M. A.; LORENZO, M. y PRIEGUE, D. (2011). "Infancia de la inmigración y educación: la visión de las familias. Revista de investigación educativa, 29 (1), 97-110.

SLAVIN, R.E. (2003). "Cooperative learning and intergroup relations", en BANKS, J. y MCGEE, C. Handbook of research on multicultural education. San Francisco: Jossey-Bass, pp. 628-634.

SORIANO, E. (2008). "Formación del profesorado para la educación intercultural", en VERA, J. (Coord.) Propuestas y experiencias de educación intercultural. Madrid: Fundación SM, pp. 57-84.

SORIANO, E. (2009). Vivir entre culturas: una nueva sociedad. Madrid: La Muralla.

STAKE, R.E. (1999). Investigación con estudio de casos. Madrid: Morata.

VALLS, R. y Otros (2002). Comunidades de aprendizaje. Transformar la educación. Barcelona: Graó. 